\title{
小檗碱衍生物合成及生理活性研究进展
}

\author{
丁阳平 ${ }^{a, b}$ 叶小利 $c$ 周 洁 $b$ \\ 罗赛 $b$ 练梦菲 $b$ 李学刚 $*, a$ \\ $\left({ }^{a}\right.$ 西南大学药学院 重庆 400716) \\ ( ${ }^{b}$ 西南大学食品学院 重庆 400716) \\ ( $c$ 西南大学生命科学院 重庆 400716)
}

\begin{abstract}
摘要 小檗碱是一种季铵型异喹啉生物碱, 主要存在于毛莨科、芸香科和小檗科等植物中, 具有多种药理功能. 近年 来, 大量小檗碱衍生物的合成, 极大提高了小糪碱的生理活性及拓展了小檗碱的应用范围. 文章重点综述了小檗碱及 其衍生物的生物及化学合成, 简述了其生理活性, 并对新型小檗碱衍生物合成的设计方向作了展望.

关键词小檗碱衍生物; 合成; 生理活性; 进展
\end{abstract}

\section{Progress in Synthesis and Physiological Activity of Berberine Derivatives}

\author{
Ding, Yangping ${ }^{a, b} \quad$ Ye, Xiaoli ${ }^{c} \quad{\text { Zhou, } \mathrm{Jie}^{b}}^{b}$ \\ Luo, Sai $^{b} \quad$ Lian, Mengfei ${ }^{b} \quad$ Li, Xuegang ${ }^{*, a}$ \\ ( ${ }^{a}$ School of Pharmaceutical Science, Southwest University, Chongqing 400716) \\ ( ${ }^{b}$ School of Food Science, Southwest University, Chongqing 400716) \\ ( ${ }^{c}$ School of Life Science, Southwest University, Chongqing 400716)
}

\begin{abstract}
Berberine, with a variety of pharmacological effects, is a quaternary ammonium salt from the group of isoquinoline alkaloids, which mainly exist in ranunculaceae, rutaceae and berberidaceae. Now some literatures report that berberine derivatives have more accordingly physiological activity than berberine and enlarge the application fields of berberine. In this paper, the biological and chemical synthesis and the biological activity of berberine and its derivatives are reviewed, as well as the prospects of a new synthetic strategy of berberine derivatives.
\end{abstract}

Keywords berberine derivative; synthesis; physiological activity; progress

小檗碱又称黄连素(Berberine, BBR), 是黄连中主 要的异喹啉生物碱, 含量高达 $5 \% \sim 8 \%$, 占黄连总生物 碱的 $40 \%$ 左右, 不仅含量最高, 且是其主要有效成分 ${ }^{[1]}$. 1826 年夏瓦利埃和佩尔坦首次从Xanthoxylonclava 树皮 中获得, 其分子式为 $\mathrm{C}_{20} \mathrm{H}_{18} \mathrm{NO}_{4}$, 分子量 336.37, 常态下 为淡黄色粉末, 加热失水后为土黄色. 在传统中医中被 长期用于治疗由细菌感染引起的胃肠道疾病. 近年来, 大量研究表明, 小檗碱还有抗心律失常、抗传染性原虫、 抗氧化、降糖、调脂、降压和强心等作用.

\section{1 小檗碱生物合成}

小檗碱的化学合成始于对小檗碱生物合成的研究. 早在 1910 年, 研究人员就提出了芐基异喹啉生物碱合 成途径的假说. 该假说认为小檗碱的合成始于 $L$-酪氨酸 ( $L$-Tyrosine), $L$-酪氨酸在体内经过酶的催化能转变为多 巴胺和 4'-羟基苯乙醛. 1963 年 Gear 和 Spenser ${ }^{[2]}$ 对北美 黄连的研究证实了 $L$-酪氨酸是小檗碱生物合成的先质, 它能形成小檗碱四个环的基本骨架. 但用标记好的多巴 胺作为原料, 合成的产物中只能检测到一分子被标记

\footnotetext{
* E-mail: barry4784088@126.com, Tel.: 13883341918

Received August 15, 2011; revised September 30, 2011; accepted November 17, 2011.

Project supported by the Natural Science Foundation of Chongqing City (Nos. 2010BB1134, 2008BB5257), the Major Technologies R\&D Program of Chongqing City (No. 2008AA5021) and the Key Project of Chongqing Science \& Technology Commission (No. 2010AC5007).

重庆市自然科学基金(Nos. 2010BB1134, 2008BB5257)、重庆市重大科技专项(No. 2008AA5021)和重庆市科委攻关(No. 2010AC5007)资助项目.
} 
物, 后经研究发现多巴胺中的苯环只能形成小檗碱的 $\mathrm{A}$ 环, 这证实了 $L$-酪氨酸在生物合成过程中除了能转变成 多巴胺外还能转变成另一重要中间体 4'-差基苯乙醛, 其中 4'-羟基苯乙醛中的苯环形成的是小檗碱 D 环. 随 后研究者又发现了一种合成关键酶, 即去甲劳丹素合成 酶(Norlaudanosoline synthase), 它既能催化多巴胺和 3',4'-二羟基苯乙醛缩合形成全去甲劳丹素 (S-Norlaudanosoline), 也能催化多巴胺和 4'-羟基苯乙醛 形成去甲基乌药碱( $S$-Norcoclaurine), 去甲基乌药碱经 过乌药碱 $N$-甲基转移酶催化, 被腺苷甲硫氨酸多次甲 基化后转变成另一个重要中间体番茄枝碱 ( $S$-Reticurine), 研究表明这一阶段的合成主要发生在细
胞质中 ${ }^{[3]}$. 而第二阶段的合成主要是在内质网的囊泡中 进行, 番茄枝碱经相应载体转运到内质网的囊泡中, 经 小檗碱桥酶(berberine bridge enzyme)催化番茄枝碱构建 $\mathrm{C}$ 环, 形成金黄紫堇碱 $(S \text {-Scoulerine })^{[4]}$, 到此为止小檗 碱的 A, B, C, D 环基本骨架已形成. 金黄紫堇碱在甲基 转移酶的作用下在 $\mathrm{C}(9)$ 位形成甲氧基转变为四氢非洲 防己碱( $S$-Tetrahydrocolumbamine), 四氢非洲防已碱在 亚甲基二氧基成环酶的作用下形成 $\mathrm{A}$ 环上特殊的亚甲 基二氧基环，后经氢化小檗碱氧化酶的氧化最终形成小 檗碱 ${ }^{[5]}$. 到目前为止, 虽然小檗碱的生物合成并不十分 清楚, 但 Scheme 1 的合成步骤已被人们广泛接受.<smiles>CCCCCCCCc1ccc(O)c(O)c1</smiles>

L-Tyrosine (2)

$p$-Hydroxyphenylacetaldehyde $(\mathbf{5})$

(S)-Norcoclaurine (6)

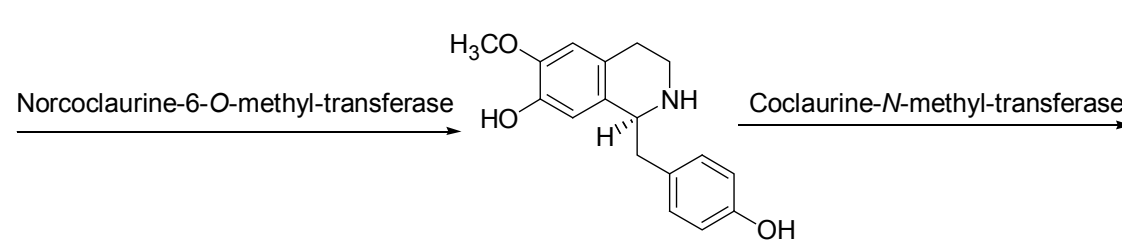

(S)-Coclaurine (7)

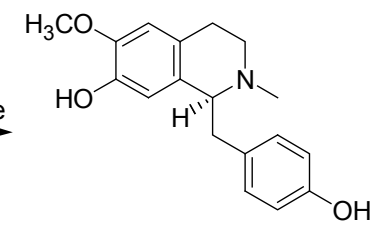

(S)-N-Methylcoclaurine (8)

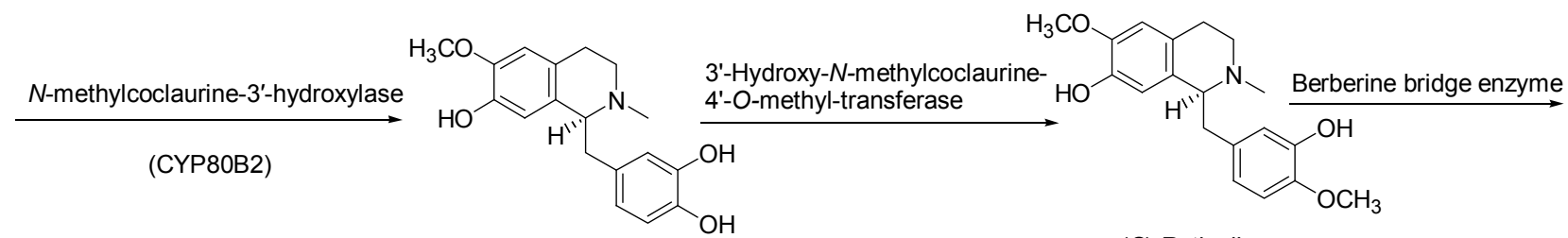

(S)-3'-hydroxy- $N$-methylcoclaurine (9)

(S)-Reticuline (10)

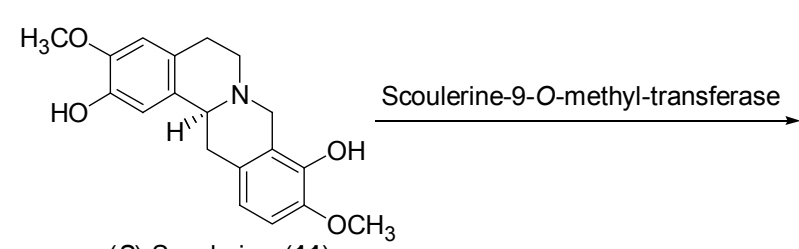

(S)-Scoulerine (11)

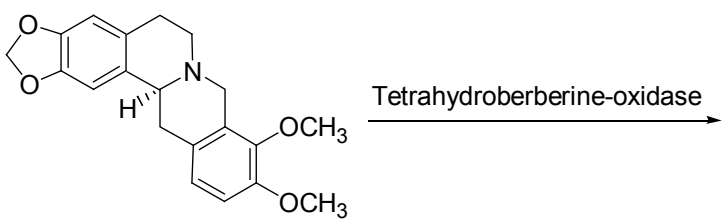

(S)-Tetrahydroberberine (13)

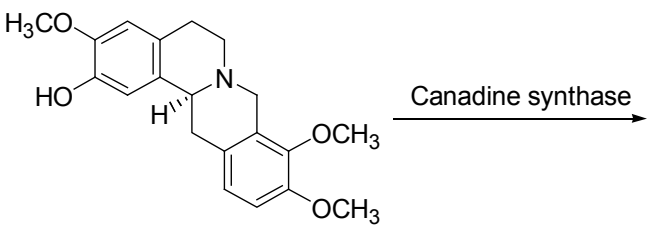

(S)-Tetrahydrocolumbamine(12)

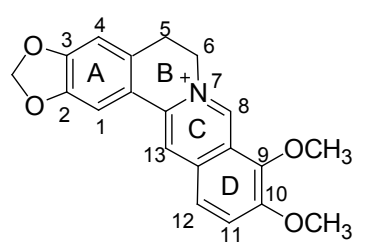

Berberine (1)

Scheme 1 


\section{2 小檗碱及衍生物的化学合成}

\section{1 小檗碱的化学合成}

小檗碱化学合成方法来源于它的生物合成, $\operatorname{Bian}^{[6]}$ 和 Yang 等 ${ }^{[7]}$ 分别以 3,4-亚甲基二氧基苯乙胺和苯甲醛 衍生物作为起始原料, 经过三步完成了小檗碱的全合成 (Scheme 2). 跟生物合成类似, 3,4-亚甲基二氧基苯乙胺 中的苯环只能形成小檗碱的 $\mathrm{A}$ 环, 而苯甲醛衍生物中的 苯环只能形成 D 环. 化合物 $\mathbf{1 4}, 15$ 首先经缩合反应生成 席夫碱 16 , 席夫碱经还原得到胺类化合物 17 , 最关键的 步骤是化合物 17 与乙二醛进行分子内环合, 最终完成 B, C 环的构建.

\section{2 小檗碱衍生物的合成}

\subsubsection{N(7)上取代衍生物的合成}

要想在 $\mathrm{N}$ 原子上进行取代反应首先要将小檗碱还 原成二氢或四氢小檗碱, 再用卤代化合物进行季铵化反 应, 生成一系列 $\mathrm{N}$ 取代衍生物 ${ }^{[8]}$ (Scheme 3). 由于季铵 化反应破环了 $\mathrm{C}$ 环的共轭体系, 大量药理实验研究表 明, 其抗菌活性降低, 但抗心血管疾病的活性增强, 当 $\mathrm{N}(7)$ 位引入烷基后抗菌活性有所增加, 但仍低于小檗碱 本身 ${ }^{[9 \sim 14]}$.

\subsection{2 小檗碱 $\mathrm{C}(8)$ 位上取代衍生物的合成}

由于小檗碱 $N(7), C(8)$ 位具有极性 ${ }^{-} N=C$ 键, 使得 $\mathrm{C}(8)$ 原子在碱性条件下很容易受到亲核试剂的进攻发 生亲核加成反应，常用的亲核试剂有 $\mathrm{OR}^{-}, \mathrm{OH}^{-}, \mathrm{R}^{-}$, $\mathrm{CCl}_{3}^{-}$及一些含 $\mathrm{N}, \mathrm{S}$ 的基团 ${ }^{[15 ~ 19]}$, 反应生成 $\mathrm{C}(8)$ 位取代
的二氢小檗碱衍生物 ${ }^{[20]}$, 这种中间体很不稳定，在酸性 条件下很容易转变为原来的季铵盐型式(Schemes 4, 5). 二氢化合物经氧化后得到相应小檗碱衍生物, 其中烷基 化衍生物能大大增强抗菌活性. Iwasa 研究组 ${ }^{[21]}$ 以盐酸 小檗碱为原料通过嗍氢化钠还原，再经格氏试剂烷基 化，最后氧化得到 C(8)位取代的烷基及芳基小檗碱衍生 物(Scheme 5), 其中烷基化衍生物的抗菌实验研究发现, 其活性强弱与碳链长度成正相关. 杨勇等 ${ }^{[22]}$ 直接用格 氏试剂对小檗碱进行烷基化, 氧化后得到一系列烷基小 檗碱(Scheme 5), 其中 8-辛基-小檗碱的抗菌活性最强, 对 B. subtilis(枯草杆菌)的抗菌活性比小檗碱提高了 125 倍. Grycova 课题组 ${ }^{[23]}$ 将小檗碱与唑类(吡咯、吡唑、咪 唑、三唑)加入到含有氢化钠的四氢呋喃中发生亲核加 成反应生成 $C(8)$ 位取代的 7,8-二氢小檗碱衍生物. Cheng 等 ${ }^{[24]}$ 以盐酸小檗碱为原料经过四步反应成功地在 $C(8)$ 位上引入两个短链烷基，该合成路线为小檗碱首先由强 碱处理生成内酰胺 $\mathbf{2 8}$, 再经三氯氧化磷氧化并卤代生 成 8-氯代小檗碱, 后经格氏试剂烷基化得 8,8-二烷基二 氢小檗碱(Scheme 6).

\subsection{3 小檗碱 C(9)位取代衍生物的合成}

大部分小檗碱 $C(9)$ 位上的取代反应都是通过小檗 红碱来完成的. 活性试验研究发现这类化合物对 G-四 连体 DNA 具有很强的亲和能力, 并且对 DNA 聚合酶具 有较强的抑制效果, 起到稳定 DNA 的作用. 小檗碱经 热解、烷基化反应能生成一系列 9- $O$-小檗碱衍生物 (Scheme 7). Iwasa 研究组 ${ }^{[10]}$ 首先利用该反应成功地将
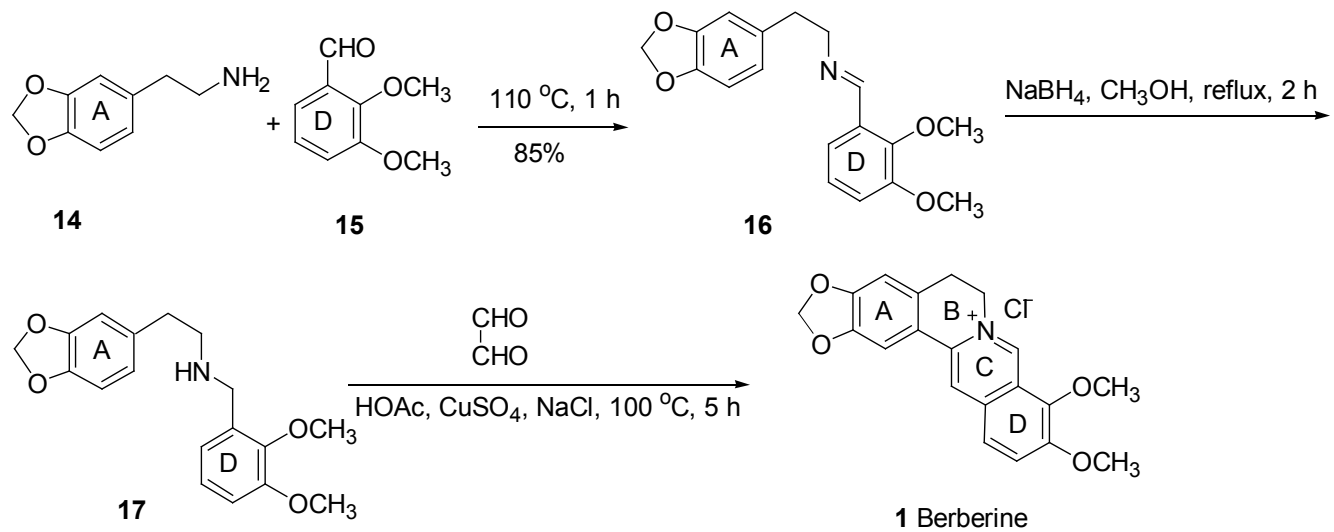

1 Berberine

Scheme 2
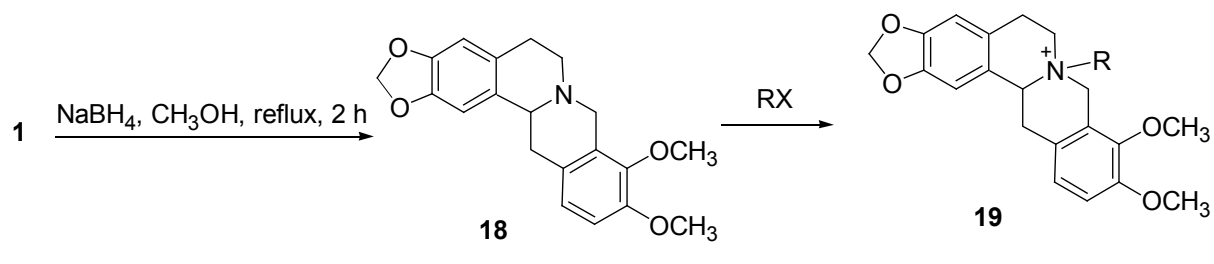

Scheme 3 


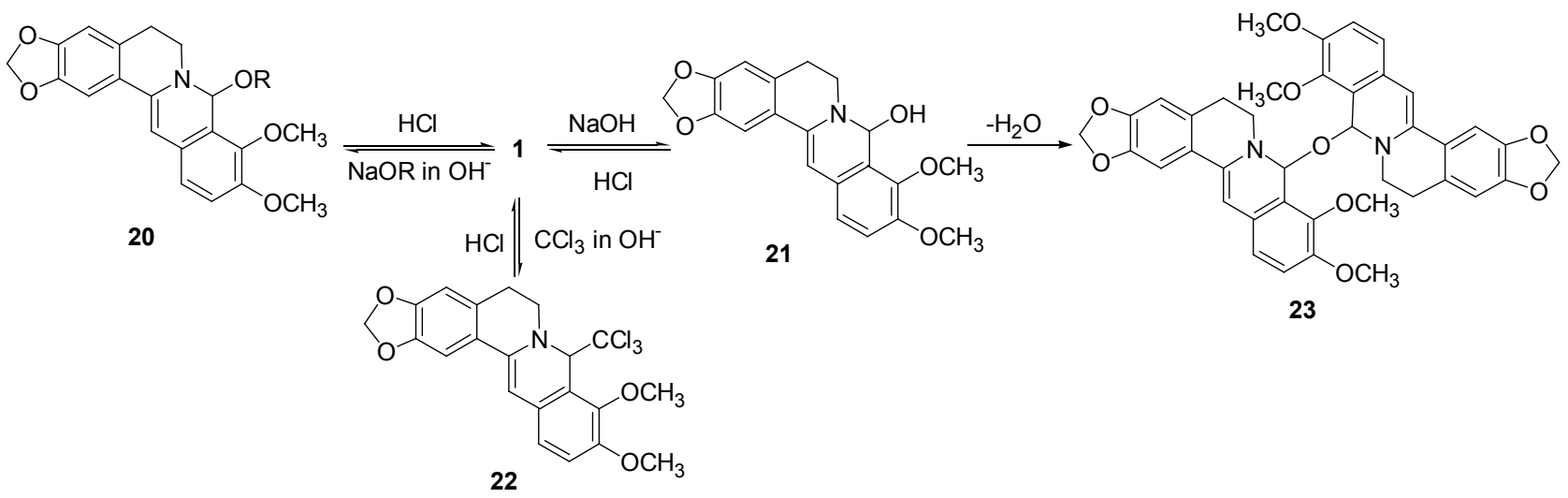

Scheme 4

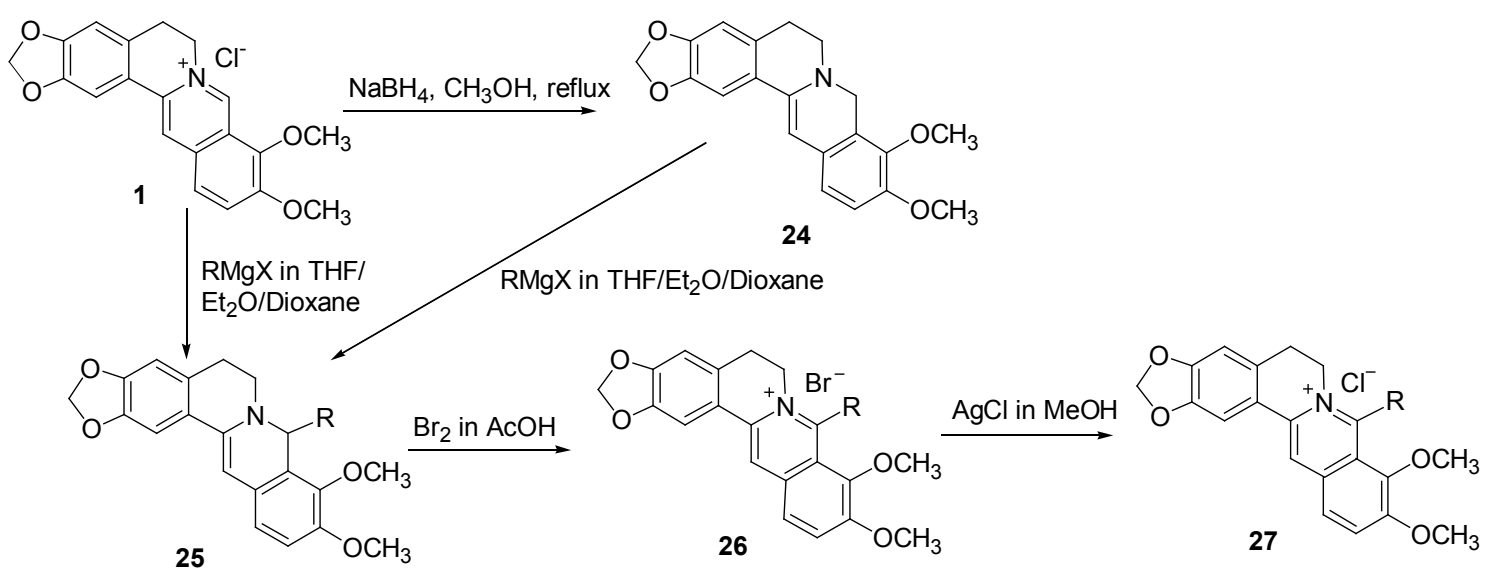

Scheme 5

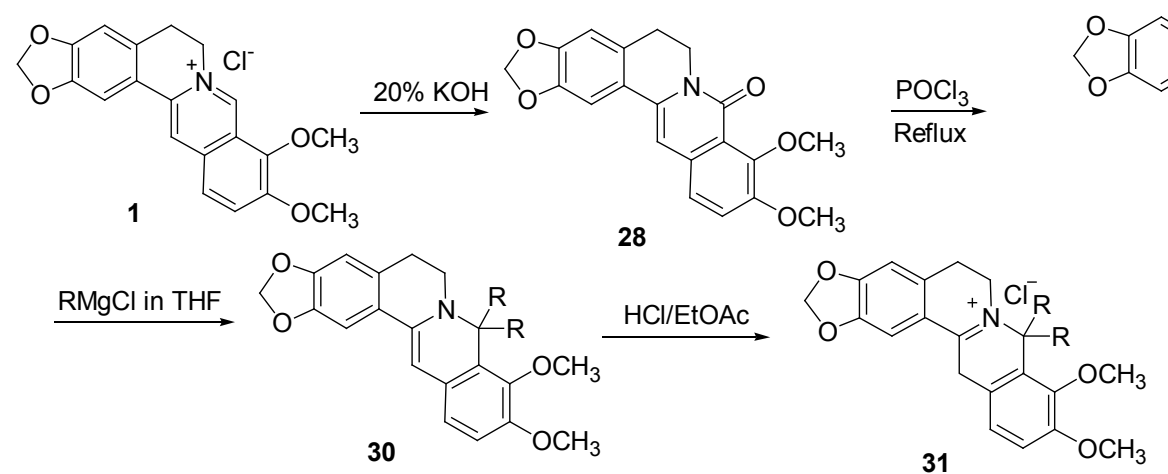

Scheme 6

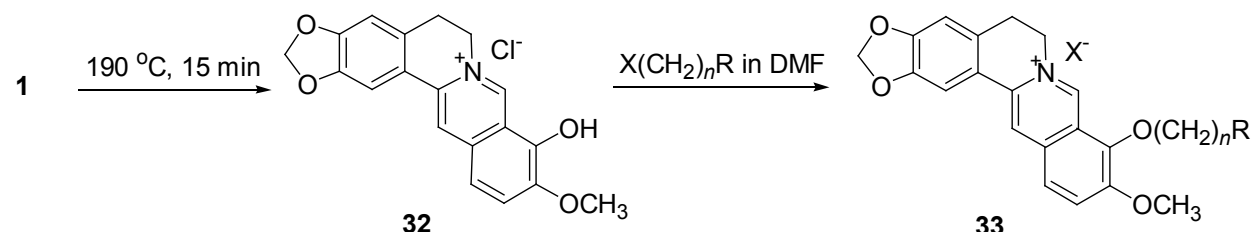

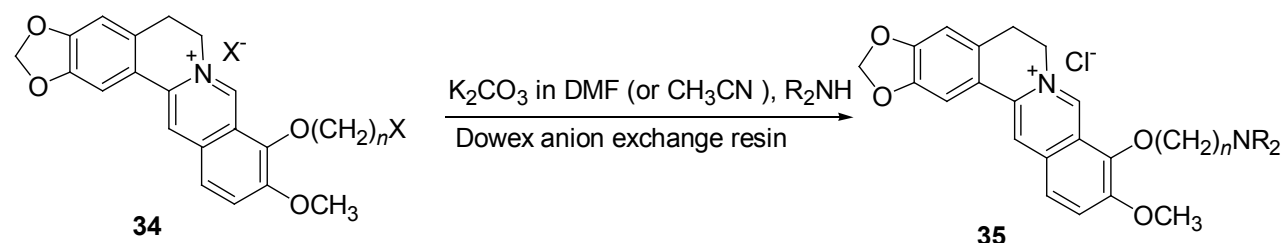

Scheme 7 
$\mathrm{C}$ (9)位甲氧基中的甲基变为乙基(33 中 $\mathrm{R}$ 为乙基). 2005 年 Pang 课题组 ${ }^{[25]}$ 设计合成了与 CT DNA 亲和力很强的 9-O-乙胺小檗碱; 同年 Chen 等 ${ }^{[26]}$ 利用两分子小檗碱成 功获得小檗红碱二聚体, 它与 DNA 的亲和力比小檗碱 提高了 100 倍; 随后 Long 等 ${ }^{[27]}$ 不仅得到小檗红碱二聚 体, 还成功合成出小檗红碱与药根碱的聚合物, 但后者 与 CT DNA 的亲和力要弱于前者; 2007 年 Zhang 等 ${ }^{[28]}$ 继续对化合物 34(此时 34 中 X 为溴) 进行氨解得到一系 列含氮侧链衍生物, 活性试验发现具有三个亚甲基和末 端氨基的化合物能明显增强与 $\mathrm{G}$ 四连体 DNA 的亲和力, 并能有效抑制 telo21 DNA 扩增及 DNA 聚合酶的活性; 同样 $\mathrm{Ma}$ 等 ${ }^{[29]}$ 在小檗红碱 $\mathrm{C}(9)$ 位羟基上成功地接上一系 列杂环化合物, 活性试验研究发现这类衍生物对 DNA 末端 $\mathrm{G}$ 四连体的亲和力具有高度选择性. Halimani 研究 组 ${ }^{[30]}$ 用叠氮化钠处理化合物 34 得到一系列叠氮化合物, 此类化合物具有很强的抗癌活性. 2010 年 $\mathrm{Li}$ 等 ${ }^{[31]}$ 研究 发现小檗红碱能很好地清除血液中总胆固醇和低密度 脂蛋白, 并利用小檗红碱酯类衍生物在体内能被一种酯 酶水解成小檗红碱的特点设计合成了一系列 C (9)位酯 类小檗碱衍生物. 同年 Huang 研究组 ${ }^{[32,33]}$ 成功地在 $\mathrm{C}(9)$ 差基上引入烷基醚类化合物, 其中 9- $O$-丁基苯甲醚对乙 酰胆碱酯酶的抑制能力最强. 2011 年 Shi 等 ${ }^{[34]}$ 通过小檗 红碱设计合成了一系列 $\mathrm{C}(9)$ 位被取代的三夾小檗碱衍 生物, 并研究了其对乙酰胆碱酷酶和丁酰胆碱酯酶的抑 制作用, 结果显示大多数三唑小檗碱衍生物都能抑制乙 酰胆碱酯酶, 并且普遍强于对丁酰胆碱酯酶的作用, 其 中 9-O-(4-异丙基胺-1-丁基)-三唑小檗碱衍生物对乙酰 胆碱酯酶抑制作用最强, 半抑制浓度为 $0.044 \mu \mathrm{mol} \cdot \mathrm{L}^{-1}$. 同年 Hardik 研究组 ${ }^{[35]}$ 发现小檗碱红碱衍生物具有抗 $\mathrm{HIV}$ 病毒作用, 只是活性比小檗碱 $\left(\mathrm{EC}_{50}=0.13 \mu \mathrm{mol} \cdot\right.$ $\mathrm{L}^{-1}$ )弱.

2008 年, Huang ${ }^{[36]}$ 和 $\mathrm{Ma}$ 等 ${ }^{[37]}$ 利用胺类化合物对小 檗碱 $\mathrm{C}(9)$ 位甲氧基直接进行氨解反应首次合成了一系 列 9- $N$-取代衍生物(Eq. 1), 活性试验研究表明该类化合 物与 $\mathrm{G}$ 四连体 DNA 的亲和力比 9-O-取代衍生物强.

1

$\underset{\text { reflux } 2 \sim 4 \mathrm{~h}}{\mathrm{NH}_{2}\left(\mathrm{CH}_{2}\right)_{n} \mathrm{R} \text {, ethanol }}$

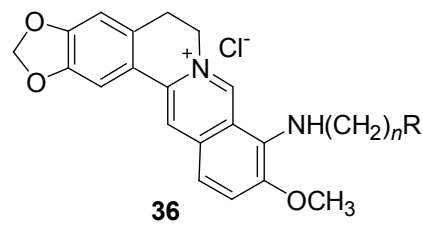

2.2.4 小檗碱 $\mathrm{C}(12)$ 位取代衍生物的合成

小檗碱 $\mathrm{C}(12)$ 位上的取代反应较少见, 到目前为止 只有 1998 年 Iwasa 研究组 ${ }^{[21]}$ 报道 8-烷基小檗碱按物质 的量的比 $1: 9$ 与溴反应生成 8-烷基-12-澳-小檗碱, 但
经过本实验室研究发现, 溴的取代位置并非 C(12)位, 而是 $\mathrm{C}(13)$ 位 $^{[38]}$.

\subsection{5 小檗碱 $\mathrm{C}(13)$ 位上的取代反应}

研究报道小檗碱 $C(13)$ 位取代衍生物具有多种生物 活性, 如抗菌、抗虐疾等. 由于小檗碱具有带正电荷的 异喹啉环，直接在 C(13)发生取代反应非常困难，因此 在合成该类衍生物时，应用较多的是利用 Mannich 反应 使小檗碱在碱性条件下与丙酮或烯丙基化合物(如烯丙 基三丁基锡、烯丙基碘化镁等)发生加成反应生成 $\alpha$-取 代烯胺中间体，此中间体再与亲电试剂卤代烷反应生成 $\mathrm{C}(13)$ 取代小檗碱衍生物, 但反应的缺点是 8-丙酮基二 氢小檗碱很容易发生消除反应变回原料，从而降低第二 步烯胺烷基化产率. 为了使烯胺烷基化达到较理想的效 果, 操作过程中应尽量避免水、酸的干扰, 反应过程中 采用氮气保护. 烯胺烷基化可能的反应机制见 Scheme 8. 8-丙酮基二氢小檗碱首先形成 $\mathbf{3 8} \mathbf{a}$ 中间体，随后通过 $[3,3]-\sigma$ 迁移重排形成 $\mathbf{3 8 b}$, 并经过逆烯反应 ${ }^{[39]}$, 生成一 系列 $\mathrm{C}(13)$ 取代小檗碱衍生物, 丙酮及烯丙基化合物在 整个过程中起的是催化作用.

1996年 Iwasa 等 ${ }^{[10]}$ 首次利用丙酮在碱性条件下获得 8-丙酮基二氢小檗碱, 再在高温条件下经碘代乙烷取代 生成 13-乙基小檗碱. Lee 等 ${ }^{[40}$ 利用相同方法获得不同碳 链的 13-烷基小檗碱. Park 等 ${ }^{[41]}$ 为了寻找抗菌活性更好 的化合物, 利用该中间体在 $\mathrm{C}(13)$ 位成功引入了一系列 芳香基团, 最终发现 13-(4-异丙基)芐基小檗碱抗菌活性 最强. 为了增强 G-四链体 DNA 的稳定性及对 DNA 聚 合酶的抑制作用, Franceschin 等 ${ }^{[42]}$ 利用 8-丙酮基二氢小 檗碱作为中间体设计合成了 13 -乙基哌啶小檗碱. Bremmer 等 ${ }^{[43]}$ 在此基础上(除了用上述方法以外), 应用 丙烯基碘化镁格氏试剂和丙烯基三丁基锡得到中间体 40, 再与卤代化合物反应生成一系列化合物 41 (Scheme 9). 同样 Ball 等 ${ }^{[44]}$ 利用丙烯基三丁基锡在小檗碱 C(13) 位引入 MDR 抑制剂 $I N F_{55}$ 得到化合物 $\mathbf{4 2}$ (Scheme 9), 该 化合物对多种细菌都具有抑制作用. Samosorn 等 ${ }^{[45]}$ 利用 甲醇和盐酸在高温下处理该中间体，得到 13-差基小檗 碱, 再与卤代化合物反应生成 C(13)位醚类衍生物, 其 中 13-芐氧基衍生物( $\left(\mathrm{INF}_{55}\right.$ 通过氧与小檗碱连接)抗菌活 性比小檗碱增加了 382 倍. $\mathrm{Li}$ 等 ${ }^{[46]}$ 成功利用 8-着基二氢 小檗碱与卤素互化物 $(\mathrm{ICl})$ 在室温下反应 $5 \mathrm{~d}$ 得到 13-碘 代小檗碱.

上述方法能较容易地在 $\mathrm{C}(13)$ 位引入各种基团, 大 大地拓展了小檗碱地开发利用, 缺点是合成产率较低 (47\% 54\%), 并且化合物 37 很容易发生消除反应转变 成原料小檗碱. 另一种方法是先用硼氢化钠直接将小檗 碱还原成二氢小檗碱, 然后用亲电试剂(如卤化物)进行 


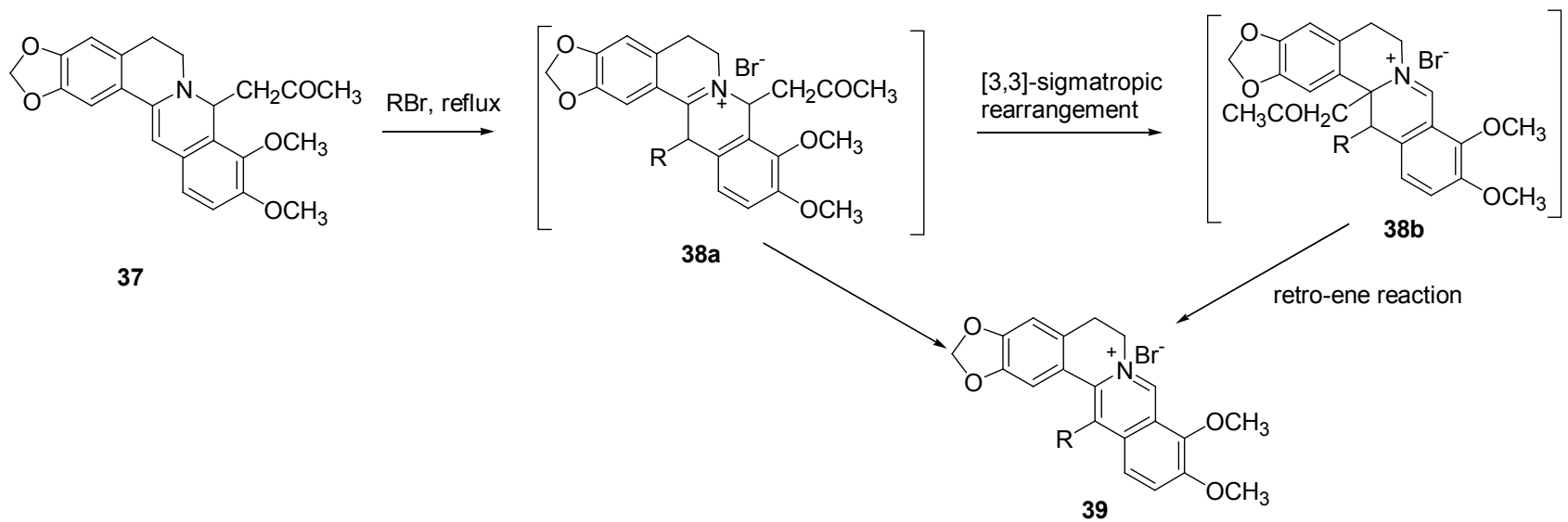

Scheme 8

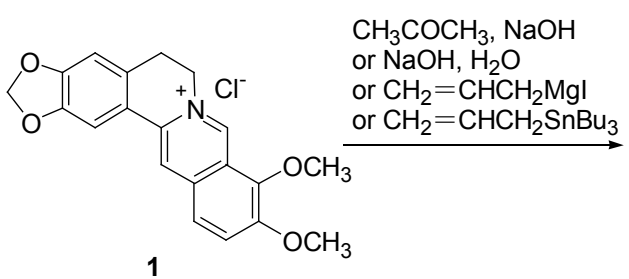<smiles>[R]CC1c2c(ccc(OC)c2OC)C2=Cc3cc4c(cc3CCN21)OCO4</smiles><smiles>[X][M]1CCc2cc3c(cc2-c2c1ccc(OC)c2OC)OCO3</smiles>
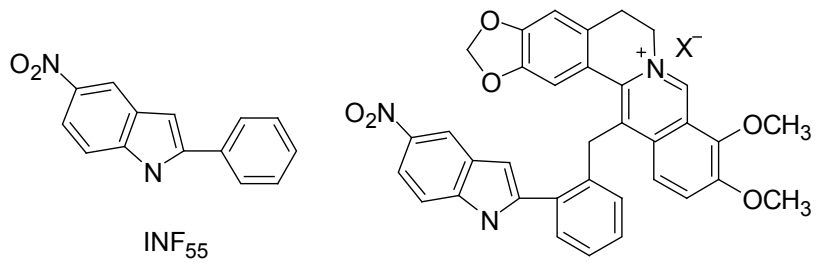

42

Scheme 9

烯胺烷基化, 经氧化后可在 C(13)位引入各种基团 (Scheme 10). 如 Jang 等 ${ }^{[47]}$ 利用该方法获得一系列 C(13) 位取代的芳香族小檗碱. Samosorn 等 ${ }^{[48]}$ 分别利用上述两 种方法合成了一系列 C(13)位酯化小檗碱. Lee 等 ${ }^{[40]}$ 利用 该方法获得 13 -乙基二氢及四氢小檗碱. $\mathrm{Li}^{[49]}$ 和 $\mathrm{Li}$ 等 ${ }^{[8]}$ 成功地用醛类化合物在小檗碱 C(13)位引入烷基. 该方 法的产率比上一种方法要高.
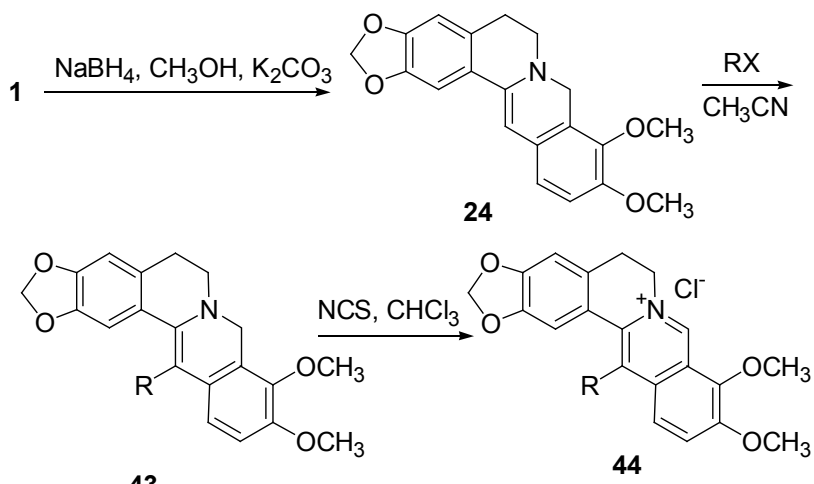

2.2.6 其它

小檗碱在路易斯酸存在的条件下能转变成巴马汀 衍生物. $\mathrm{Hu}^{[50]}$ 和 $\mathrm{Li}$ 等 ${ }^{[31]}$ 应用间苯三酚成功打开 2,3-亚甲 基二氧基环，并脱掉它们的亚甲基，但对 $\mathrm{C}(9), \mathrm{C}(10)$ 位 甲氧基不起作用，生成一系列 2,3-二羟基巴马汀衍生物 (Scheme 11). Beausoleil 研究组 ${ }^{[51]}$ 利用三溴化硼使 2,3 -亚 甲基二氧基开环，并能脱掉所有甲氧基中的甲基生成多 酚类巴马汀衍生物, 而三氯化硼与间苯三酚的作用相似 (Scheme 11). Lee 等 ${ }^{[40]}$ 将 C(13)位取代小檗碱放在含有路 易斯酸 $\left(\mathrm{AlCl}_{3}\right)$ 的甲苯中回流能生成 2,3,9-三差基巴马汀 衍生物(Scheme 11).

$\mathrm{Jia}^{[52]}$ 和 Weng 等 ${ }^{[53]}$ 用有机酸和盐处理小檗碱得到 离子对化合物 48(小檗碱脂肪族有机酸盐)(Eq. 2), 此类 化合物对心律不齐、血小板聚集、高血压、消化性溃疡 和糖尿病有特殊疗效.

Huang 等 ${ }^{[54]}$ 利用二氨基化合物及 $N, N$-二甲基胺类 化合物在乙醇中回流得到化合物 49 (Eq. 3).

Scheme 10 


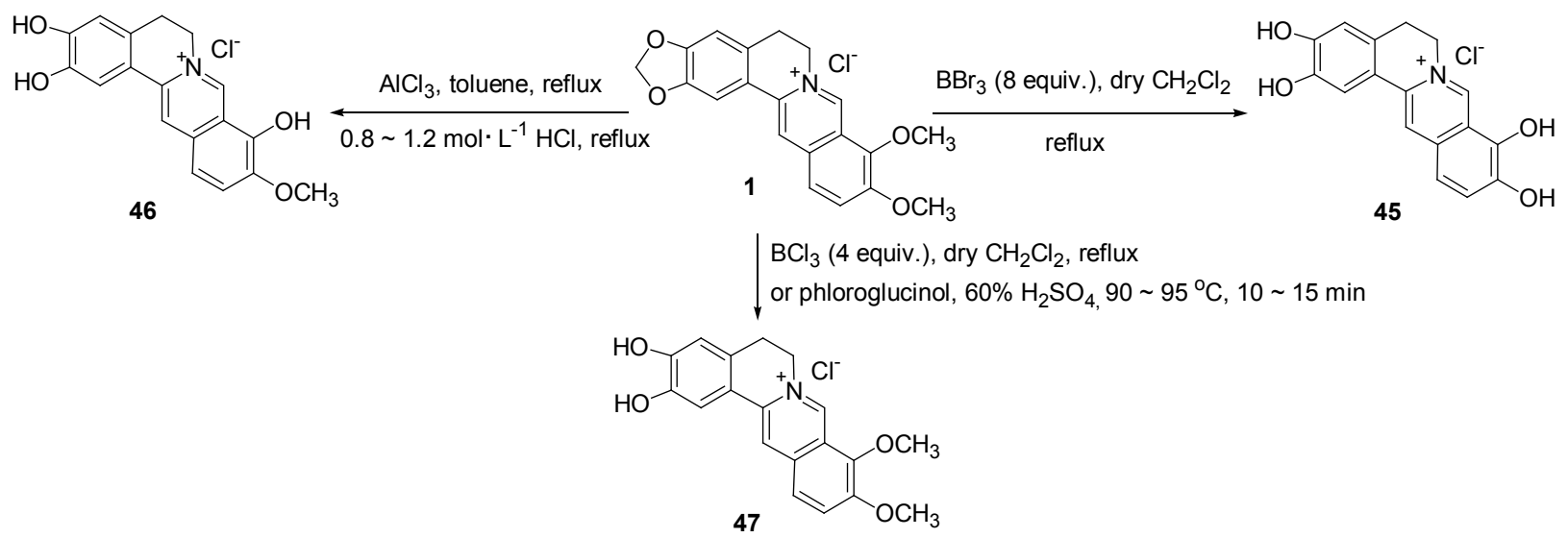

Scheme 11

$\mathrm{CH}_{3}\left(\mathrm{CH}_{2}\right)_{10} \mathrm{COOH}, \mathrm{NaHSO}_{4}$

$\mathrm{H}_{2} \mathrm{O}, 4 \mathrm{~h}, 80^{\circ} \mathrm{C}$
$\mathrm{CH}_{3}\left(\mathrm{CH}_{2}\right)_{10} \mathrm{COO}^{-} \quad \mathrm{OCH}_{3}$<smiles>COc1ccc2cc3[n+](cc2c1C)CCc1cc2c(cc1-3)OCO2</smiles>

(2)

(3)

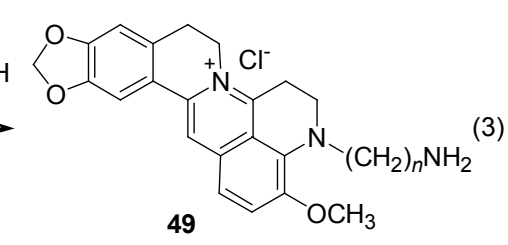

Park 研究组 ${ }^{[55]}$ 设计合成了一系列 C(9), C(13)同时 取代的小檗碱衍生物, 并研究了其抑菌活性, 研究结果 表明, 引入烷基能增强其抗菌活性, 而酰基则会使其活 性降低. 其中 9- $O$-丁基-13-(4-异丙基芐基)-小檗碱的活 性最强, 对新型隐球菌和念球菌属的 MIC (minimum inhibitory concentration)为 $0.25 \sim 1 \mu \mathrm{g} / \mathrm{mL}$, 对曲霉属真 菌达到 $2 \sim 4 \mu \mathrm{g} / \mathrm{mL}$.

Bahar 研究组 ${ }^{[56]}$ 用碱处理小檗碱得到化合物 50, 再 与 $\mathrm{POCl}_{3}$ 反应得到 8 -氯代一小檗碱, 氯代小檗碱与格氏 试剂进行偶合反应成功地在 $\mathrm{C}(8)$ 引入两个烷基, 最后经 氧化及芳构化反应得到目标产物 52 (Scheme 12). 活性 试验发现化合物 52 对布氏冈比亚雉虫的 $\mathrm{IC}_{50}$ 值为 $0.0091 \mu \mathrm{mol} \cdot \mathrm{L}^{-1}$, 强于阳性对照药苏拉明 $\left(\mathrm{IC}_{50}\right.$ 为 0.25 $\left.\mu \mathrm{mol} \cdot \mathrm{L}^{-1}\right)$.

\section{3 展望}

综上所述, 小檗碱具有多种生理活性, 当引入特定 基团后能大大增强其相应活性. 为了进一步开发活性更 强的小檗碱衍生物, 现将具有抑菌、降糖、抗肿瘤及抗 氧化新型小檗碱衍生物的合成设计思路归纳如下.

抑菌衍生物的设计思路: 资料显示在小檗碱 $\mathrm{C}(8)$,<smiles>COc1ccc2cc3n(c(=O)c2c1OC)CCc1cc2c(cc1-3)OCO2</smiles><smiles></smiles>

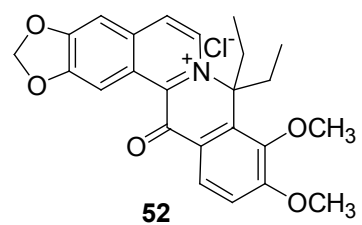

\section{Scheme 12}

$\mathrm{C}(9)$ 及 $\mathrm{C}(13)$ 位上分别引入长链烷基(如辛基)能大大提 高小檗碱的抗菌活性, 由此设想在 $C(8), C(9)$ 和 $C(13)$ 两 两或同时引入烷基是否具有协同增效作用. 因此此类化 合物是小檗碱衍生物抗菌活性的设计方向.

降糖衍生物的设计思路: 研究发现, 一般抗菌活性 很强的化合物具有较强的毒性, 因此在开发降糖衍生物 时一般不选择引入上述的烷基链，再者烷基衍生物会降 低水溶性, 可能会降低生物利用度. 资料显示 $\mathrm{C}(2), \mathrm{C}(3)$ 位的亚甲二氧基环是 $\mathrm{BBR}$ 降糖的必须基团, 而芳 $\mathrm{C}$ 环 结构并非必需，再者还原后的二氢及四氢小檗碱毒性降 低，因此降低毒性、提高细胞对葡萄糖的消耗及生物利 用率是开发降糖衍生物的关键. 因此二氢及四氢小檗碱 衍生物是降糖活性的开发方向.

抗癌衍生物的设计思路: BBR 抗癌与抗菌具有一定 相关性，因此同时在 C(8), C(13)引入烷基和芳基也是研 究抗肿瘤主要方向. 另外有研究报道 C(9)位酯化衍生 
物、13-烷基小檗红碱及其酯化衍生物具有很好的抗肿 瘤作用. 因此, 在 $\mathrm{C}(8), \mathrm{C}(9), \mathrm{C}(13)$ 同时接入烷基或酰基 (如 $C(9)$ 位)也是抗肿瘤化合物的设计方向.

抗氧化衍生物的设计思路: 资料显示小檗碱具有较 强的抗氧化性能 ${ }^{[57]}$, 为了进一步开发小檗碱的抗氧化 作用, 部分或全部脱掉小檗碱的甲基或亚甲基是小檗碱 抗氧化的研究方向, 而 8-烷基多酚类小檗碱衍生物是油 脂抗氧化剂的最佳选择.

\section{References}

[1] Pu, S. B.; Xu, X. H.; Zhang, Y. H.; Zhou, X. L. Chin. Trad. Herb. Drugs 1999, 30(5), 375 (in Chinese). (兴社班, 许翔鸿, 张宇和, 周雪林, 中草药, 1999, 30(5), 375.)

[2] Gear, J. R.; Spenser, I. D. Can. J. Chem. 1963, 41, 783.

[3] Hashimoto T.; Yamada, Y. Annu. Rev. Plant Physiol. Mol. Biol. 1994, 45, 257.

[4] Steffens, P.; Nagakura, N.; Zenk, M. H. Tetrahedron Lett. 1984, 25, 951.

[5] Zenk, M. H. In The Chemistry and Biology of Isoquinoline Alkaloids, Eds.: Phillison, J. D.; Robert, M. F.; Zenk, M. H., Springer-Verlag, Berlin, 1985, p. 240.

[6] Bian, X. L.; He, L. C.; Yang, G. D. Bioorg. Med. Chem. Lett. 2006, $16,1380$.

[7] Yang, P.; Song, D. Q.; Li, Y. H.; Kong, W. J.; Wang, Y. X.; Gao, L. M.; Liu, S. Y.; Cao, R. Q.; Jiang, J. D. Bioorg. Med. Chem. Lett. 2008, 18, 4675 .

[8] Li, Y. H.; Yang, P.; Kong, W. J.; Wang, Y. X.; Hu, C. Q.; Zuo, Z. Y. J. Med. Chem. 2009, 52(2), 492.

[9] Huang, Z. Y.; Hong, M. H.; Peng, S. X.; Shi, Y. Q. J. Chin. Pharm. Univ. 1988, 19(4), 249 (in Chinese).

(黄枕亚, 冯玫华, 彭司勋, 史益强, 中国药科大学学报, 1988, 19(4), 249.)

[10] Iwasa, K.; Kamigauchi, M.; Uek, M.; Taniguch, M. Eur. J. Med. Chem. 1996, 31, 469.

[11] Wang, R. F.; Xu, G. Y.; Hua, W. Y. Chin. J. Med. Chem. 1996, 12(22), 243 (in Chinese).

(王瑞芳, 许国友, 华维一, 中国药物化学杂志, 1996, 12(22), 243.)

[12] Zhang, C.; Shen, W. B.; Huang, W. L. J. Chin. Pharm. Univ. 2002, $33(1), 70$ (in Chinese).

(张灿, 沈文斌, 黄文龙, 中国药科大学学报, 2002, 33(1), 70.)

[13] Zhang, C.; Huang, W. L. J. Chin. Pharm. Univ. 2003, 34(1), 7 (in Chinese).

(张灿, 黄文龙, 中国药科大学学报, 2003, 34(1), 7.)

[14] Zhang, C.; Lin, Y.; Hua, W. Y. J. Chin. Pharm. Univ. 2004, 35(5), 393 (in Chinese).

(张灿, 林云, 华维一, 中国药科大学学报, 2004, 35(5), 393.)

[15] Marek, R.; Sekářová, P.; Hulová, D.; Marek, J.; Dostál, J.; Sklenáŕ, V. J. Nat. Prod. 2003, 66, 481.

[16] Man, S.; Dostál, J.; Nečas, M.; Žák, Z.; Potáček, M. Heterocycl. Commun. 2001, 7, 243.

[17] Naruto, S.; Mizuta, H.; Nishimura, H. Tetrahedron Lett. 1976, 19, 1597.

[18] Shamma, M.; Rahimizadech, M. J. Nat. Prod. 1986, 49, 398.

[19] Möhrle, H.; Biegholdt, M. Arch. Pharm. 1982, 315, 919.

[20] Yu, C.; Zhou, J. Z.; Pan, J. F.; Wang, Y. P.; Jia, J. Y.; Lu, C.; Liu, G. Y.; Li, S. J.; Zhou, H. J.; Liu, Y.; Zhang, M. Q.; Liu, Y. M.; Wang, W.; Zhang, Z. C. CN 101323613, 2008 [Chem. Abstr. 2008,
$150,98510]$.

[21] Iwasa, K.; Lee, D. U.; Kaug, S. I.; Wiegrebe, W. J. Nat. Prod. 1998, 61(9), 1150.

[22] Yang, Y.; Ye, X. L.; Li, X. G.; Zhen, J.; Zhang, B. S.; Yuan, L. J. Planta Med. 2007, 73, 602.

[23] Grycova, L.; Hulova, D.; Maier, L.; Standara, S.; Necas, M.; Lemiere, F.; Kares, R.; Dostal, J.; Marek, R. Magn. Reson. Chem. 2008, 46(12), 1127.

[24] Cheng, Z.; Chen, A. F.; Wu, F.; Sheng, L.; Zhang, H. K.; Gu, M.; Li, Y. Y.; Zhang, L. N.; Hu, L. H.; Li, J. Y.; Li, J. Bioorg. Med. Chem. 2010, 18, 5915.

[25] Pang, J. Y.; Qin, Y.; Chen, W. H.; Luo, G. A.; Jiang, Z. H. Bioorg Med. Chem. 2005, 13, 5835.

[26] Chen, W. H.; Pang, J. Y.; Qin, Y.; Peng, Q.; Cai, Z. W.; Jiang, Z. H. Bioorg. Med. Chem. Lett. 2005, 15, 2689.

[27] Long, Y. H.; Bai, L. P.; Qin, Y.; Pang, J. Y.; Chen, W. H.; Cai, Z. W.; Xua, Z. L.; Jiang, Z. H. Bioorg. Med. Chem. 2006, 14, 4670.

[28] Zhang, W. J.; Ou, T. M.; Lu, Y. J.; Huang, Y. Y.; Wu, W. B.; Huang, Z. S.; Zhou, J. L.; Wong, K. Y.; Gu, L. Q. Bioorg. Med. Chem. 2007, 15, 5493.

[29] Ma, Y.; Ou, T. M.; Tan, J. H.; Hou, J. Q.; Huang, S. L.; Gu, Li. Q.; Huang, Z. S. Bioorg. Med. Chem. Lett. 2009, 19, 3414.

[30] Halimani, M.; Chandran, S. P.; Kashyap, S.; Jadhav, V. M.; Prasad, B. L.; Hatha, S.; Maiti, S. Langmuir 2009, 25(4), 2339.

[31] Li, Y. H.; Yi, L.; Yang, P.; Kong, W. J.; You, X. F.; Ren, G.; Deng, H. B.; Wang, Y. M.; Wang, Y. X.; Jiang, J. D.; Song, D. Q. Bioorg. Med. Chem. 2010, 18, 1267.

[32] Huang, L.; Shi, A. D.; He, F.; Li, X. S. Bioorg. Med. Chem. 2010, $18,1244$.

[33] Huang, L.; Luo, Z. H.; He, F.; Lu, J.; Li, X. S. Bioorg. Med. Chem. 2010, 18,4475 .

[34] Shi, A. D.; Huang, L.; Lu, C. J.; He, F.; Li, X. S. Bioorg. Med. Chem. 2011, 19, 2298.

[35] Hardik, S. B.; Sudeep, S.; Debashis, M.; Kamlesh, K. B.; Inder, P. S. Eur. J. Med. Chem. 2011, 46, 1045.

[36] Huang, Z. S.; Gu, L. Q.; Ma, Y.; Ou, T. M.; Hou, J. Q. CN 101255158, 2008 [Chem. Abstr. 2008, 149, 378915].

[37] Ma, Y.; Ou, T. M.; Hou, J. Q.; Lu, Y. J. Bioorg. Med. Chem. 2008, 16(16), 7582.

[38] Ding, Y. P. Ph.D. Dissertation, South-West University, Chongqing, 2011 (in Chinese). (丁阳平, 博士论文, 西南大学, 重庆, 2011.)

[39] Eto, M.; Nishimoto, S.; Kubota, T.; Matsuoka, K.; Harano, K. Tetrahedron 1996, 37, 2445.

[40] Lee, G. E.; Lee, H. S.; Lee, S. D.; Kim, J. H.; Kim, W. K.; Kim, Y. C. Bioorg. Med. Chem. Lett. 2009, 19, 954.

[41] Park, K. D.; Lee, J. H.; Kim, S. H.; Kang, T. H.; Moon, J. S.; Kim, S. U. Bioorg. Med. Chem. Lett. 2006, 16, 3913.

[42] Franceschin, M.; Rossetti, L. G.; D'Ambrosio, A.; Schirripa, S.; Bianco, A.; Ortaggi, G.; Savino, M.; Schultesf, C.; Neidlef, S. Bioorg. Med. Chem. Lett. 2006, 16, 1707.

[43] Bremner, J. B.; Samosorn, S. Aust. J. Chem. 2003, 56, 871.

[44] Ball, A. R.; Casadei, G.; Samosorn, S.; Bremner, J. B.; Ausubel, F. M.; Moy, T. I.; Kim, L. ACS Chem. Biol. 2006, 1(9), 594.

[45] Samosorn, S.; Tanwirat, B.; Muhamad, N.; Casadei, G.; Tomkiewicz, D.; Kim, L.; Suksamrarn, A.; Therdsak, P.; Karina, C. G.; Beck, J. L.; Bremner, J. B. Bioorg. Med. Chem. 2009, 17, 3866.

[46] Li, Z. J.; Wei, Y.; Chu, T. W.; Wang, X. Y.; Liu, X. Q.; Wang, Y.; Hu, S. W. J. Radioanal. Nucl. Chem. 2005, 265(3), 355.

[47] Jang, M. J.; Jwa, M.; Kim, J. H.; Song, K. J. Biol. Chem. 2002, 277(14), 12388.

[48] Samosorn, S. Ph.D. Dissertation, University of Wollongong, Wol- 
longong, 2005.

[49] Li, N. S.; Wang, H. F. CN 1629160, 2005 [Chem. Abstr. 2006, 144, 129142].

[50] Hu, L. H.; Ye, J. M.; Li, J. Y.; James, D. E. WO 2008040192, 2007 [Chem. Abstr. 2008, 148, 449809]

[51] Beausoleil, E.; Chauvignac, C.; Taverne, T. Bioorg. Med. Chem. Lett. 2009, 19, 5594.

[52] Jia, B. Z. CN 1990487, 2007 [Chem. Abstr. 2007, 147, 212106].

[53] Weng, W. Y.; Huang, J. M.; Qiu, Z. B.; Shen, T.; Zhang, J. F.; Xu, H. N.; Guo, J. X. CN 101113149, 2008 [Chem. Abstr. 2008, 148,
285384].

[54] Ma, Y.; Ou, T. M.; Tan, J. H.; Hou, J. Q.; Huang, S. L.; Gu, L. Q.; Huang, Z. S. Eur. J. Med. Chem. 2011, 46, 1906.

[55] Park, K. D.; Cho, S. J.; Moon, J. S.; Kim, S. U. Bioorg. Med. Chem. Lett. 2010, 20, 6551.

[56] Bahar, M.; Deng, Y.; Zhu, X. H.; He, S. S.; Pandharkar, T.; Drew, M. E. Bioorg. Med. Chem. Lett. 2011, 21, 2606.

[57] Chen, J. J.; Chang, Y. L.; Teng, C. M.; Chen, I. S. Planta Med. 2001, 67, 593. 